\title{
Maternal protein restriction impairs the transcriptional metabolic flexibility of skeletal muscle in adult rat offspring
}

\author{
Raquel da Silva Aragão ${ }^{1,2,3}$, Omar Guzmán-Quevedo ${ }^{1,2}$, Georgina Pérez-García ${ }^{1,2}$, \\ Raul Manhães-de-Castro ${ }^{3}$ and Francisco Bolaños-Jiménez ${ }^{1,2 *}$ \\ ${ }^{1}$ INRA, CHU - HôDieu, HNB1 - UMR PhAN 1280 Physiologie des Adaptations Nutritionnelles, Place Alexis Ricordeau, \\ 44096 Nantes Cedex 1, France \\ ${ }^{2}$ Université de Nantes, Nantes Atlantique Université, Nantes, France \\ ${ }^{3}$ Departamento de Nutrição, Centro de Ciências da Saúde, Universidade Federal de Pernambuco, Recife, \\ Pernambuco, Brazil
}

(Submitted 16 April 2013 - Final revision received 29 January 2014 - Accepted 21 February 2014 - First published online 14 May 2014)

\section{Abstract}

Skeletal muscle exhibits a remarkable flexibility in the usage of fuel in response to the nutrient intake and energy demands of the organism. In fact, increased physical activity and fasting trigger a transcriptional programme in skeletal muscle cells leading to a switch from carbohydrate to lipid oxidation. Impaired metabolic flexibility has been reported to be associated with obesity and type 2 diabetes, but it is not known whether the disability to adapt to metabolic demands is a cause or a consequence of these pathological conditions. Inasmuch as a poor nutritional environment during early life is a predisposing factor for the development of metabolic diseases in adulthood, in the present study, we aimed to determine the long-term effects of maternal malnutrition on the metabolic flexibility of offspring skeletal muscle. To this end, the transcriptional responses of the soleus and extensor digitorum longus muscles to fasting were evaluated in adult rats born to dams fed a control (17\% protein) or a low-protein ( $8 \%$ protein, protein restricted $(\mathrm{PR})$ ) diet throughout pregnancy and lactation. With the exception of reduced body weight and reduced plasma concentrations of TAG, PR rats exhibited a metabolic profile that was the same as that of the control rats. In the fed state, PR rats exhibited an enhanced expression of key regulatory genes of fatty acid oxidation including $C P T 1 a, P G C-1 \alpha, U C P 3$ and $P P A R \alpha$ and an impaired expression of genes that increase the capacity for fat oxidation in response to fasting. These results suggest that impaired metabolic inflexibility precedes and may contribute to the development of metabolic disorders associated with early malnutrition.

Key words: Metabolic flexibility: Skeletal muscle: Metabolic programming

Skeletal muscle has a remarkable ability to adapt its metabolism in response to the nutrient intake and energy demands of the organism. In fact, both food deprivation and high-fat diet feeding favour the use of lipids by skeletal muscle as an energy source at the expense of glucose, a phenomenon known as metabolic flexibility. Impaired metabolic flexibility has been shown to be associated with obesity and insulin resistance ${ }^{(1,2)}$, but the exact mechanisms linking metabolic inflexibility to these pathological conditions are not known. It is also not known whether the lack of adaptation to metabolic demands is a cause or a consequence of obesity or diabetes.

It is now widely accepted that malnutrition during early life is an important predisposing factor for the development of obesity and metabolic diseases. Indeed, based on epidemiological studies demonstrating that people born with a low birth weight are at a high risk of developing the metabolic syndrome in adulthood, Hales \& Barker $^{(3)}$ proposed that in a developing organism exposed to nutrient deficiency, there is a selective distribution of nutrients to preserve brain growth at the expense of other organs. This results in the 'programming' of energy metabolism to promote survival under conditions of food restriction. However, when the adverse nutritional environment disappears, the physiological incompatibility between the 'thrifty' metabolic programming and the new nutritional conditions leads to obesity, impaired glucose tolerance, hypertension and atherosclerosis ${ }^{(3)}$

Experimental studies in different animal species have provided major support for the hypothesis proposed by Hales

Abbreviations: AMPK, AMP-activated protein kinase; $C$, control; $C P T 1 a$, carnitine palmitoyltransferase $1 \mathrm{a}$; $C_{\mathrm{t}}$, threshold cycle; EDL, extensor digitorum longus; $M C A D$, medium-chain acyl coenzyme A dehydrogenase; $P D K 4$, pyruvate dehydrogenase kinase, isozyme 4; $P G C-1 \alpha$, PPAR-gamma, co-activator $1 \alpha$; PR, protein restricted; UCP3, uncoupling protein 3. 
\& Barker $^{(3)}$ and have led to the identification of several cellular and physiological adaptive responses as potential underlying mechanisms of metabolic programming including alterations in skeletal muscle. Notably, it has been shown that reduced nutrient supply during the last $3 \mathrm{~d}$ of gestation in rats leads to decreased ATP production and decreased glucose transport and glycogen biosynthesis in skeletal muscle ${ }^{(4)}$. It has also been shown that offspring born to dams fed a low-protein diet during gestation and lactation initially exhibit better glucose tolerance and enhanced insulin sensitivity in the soleus muscle ${ }^{(5)}$, a skeletal muscle containing a high percentage of type I fibres, but become insulin resistant later in life ${ }^{(6)}$. In addition to these functional alterations, a decrease ${ }^{(7)}$, an increase in $^{(8)}$ or no change $^{(9)}$ in the number of type II fibres has been documented in rats following perinatal undernutrition. In contrast to type I fibres, which have high oxidative capacity, type II fibres posses high glycolytic activity and their density is increased in obese and insulin-resistant patients.

The overall objective of the present study was to define the consequences of protein restriction during gestation and lactation on the metabolic flexibility of skeletal muscle in adulthood. Our working hypothesis was that nutritional restriction during the critical period of perinatal development impairs the ability of skeletal muscle to adapt to the use of energy substrates (lipids and glucose) in response to the nutritional and energy demands of the body. Specifically, we examined the influence of protein restriction during gestation and lactation on the expression of a set of genes involved in the starvation-induced transcriptional programme leading to a switch in fuel usage in skeletal muscle from glucose to fat. The analysed genes included the nuclear receptors PPAR $\alpha$ and $P P A R \delta$ and the co-regulator of transcription PPARgamma, co-activator $1 \alpha(P G C-1 \alpha)$, as well as several genes that play a key role in fatty acid oxidation (carnitine palmitoyltransferase 1a (CPT1a), uncoupling protein 3 (UCP3), pyruvate dehydrogenase kinase, isozyme 4 (PDK4) and medium-chain acyl coenzyme A dehydrogenase (MCAD)). The gene myosin heavy chain class 7 (Myb7), a typical marker of slow-twitch (oxidative) fibres, was also analysed.

\section{Materials and methods}

\section{Animals and experimental manipulations}

We used a typical model of metabolic programming, which has been described in detail elsewhere ${ }^{(10)}$. In brief, virgin female Wistar rats weighing 200-220 g were obtained from Charles River and mated with 3-month-old males of the same strain. After confirmation of mating, pregnant rats were housed individually and fed ad libitum during gestation and lactation either a control diet ( $170 \mathrm{~g}$ protein $/ \mathrm{kg}$ ) or an isoenergetic lowprotein diet ( $80 \mathrm{~g}$ protein $/ \mathrm{kg}$ ). The diets were kindly provided by the Department of Nutrition of the Federal University of Pernambuco (Brazil), and their composition has been described previously ${ }^{(8)}$. All the experiments were carried out in accordance with the European Communities Council Directive of 24 November 1986 (86/609/EEC) and the Principles of Laboratory Animal Care (NIH publication no. 85-23, revised 1985) and were approved by the Ethical Committee for Animal Experimentation of the Pays de la Loire (protocol CEEA.2012.15). At $1 \mathrm{~d}$ after delivery, litter size was adjusted at random to eight pups per dam in both experimental groups maintaining an equal sex ratio. At weaning $(21 \mathrm{~d})$, female pups were excluded from the study to yield a final sample size of four male pups per dam. All pups from the control (C) and protein-restricted (PR) groups were fed standard chow until the end of the experiment. The body weight of the pups was recorded every $3 \mathrm{~d}$ during lactation and every week after weaning.

\section{Killing and tissue sampling}

At 4 months of age, rats were killed under ad libitum feeding conditions or after a fasting period of $48 \mathrm{~h}$. The soleus and extensor digitorum longus (EDL) muscles from both legs were carefully dissected under sodium pentobarbital anaesthesia $\left(50 \mathrm{mg} / \mathrm{kg}\right.$ ), immediately frozen in liquid $\mathrm{N}_{2}$ and kept at $-80^{\circ} \mathrm{C}$ until analysis. The rats were then killed by cervical dislocation. All rats were killed within the first hour of the light cycle to avoid the bias induced by the circadian variations in gene expression. Bilateral fat depots from three regions (inguinal, retroperitoneal and epididymal) as well as the mesenteric fat pad were dissected, weighed and pooled to obtain a measure of body fat, referred to as abdominal fat herein. At the end of the experimental period, a total of twelve dams (six controls and six PR) were selected for analysis. From each control and PR litter, two pups were selected at random to obtain a total of twelve control pups and twelve PR pups. From each group, six pups were killed under ad libitum feeding conditions and the other six after fasting. The muscle samples collected from the right and left legs were alternatively used for the determination of gene expression by real-time quantitative PCR and for Western blot analysis. Care was taken to allocate the two pups of the same dam to different experimental groups (fed ad libitum or fasted before killing), such that the presented results corresponded to the combined data from six animals born to six different dams.

\section{Hormone and metabolite measurements}

Insulin resistance was evaluated $15 \mathrm{~d}$ before killing by the glycaemic response to an oral glucose tolerance test after an overnight fast of $16 \mathrm{~h}$. In brief, a drop of blood was collected immediately before the administration of glucose and 20, 40, 80 , and $120 \mathrm{~min}$ after administration by severing the tip of the tail. Blood glucose concentrations were determined using a blood glucose monitor (Accu-Check ${ }^{\circledR}$ Active, Roche Diagnostics). To determine the glucose disappearance rate $\left(K_{\mathrm{G}}\right)$, the AUC was calculated using GraphPad Prism 5.0 (GraphPad Software, Inc.), and the $y$ value of the starting data point was considered to be the baseline value. Plasma from trunk blood collected at the time of killing was assayed for insulin and leptin using assay kits obtained from Millipore. Enzymatic diagnostic kits obtained from BioMérieux were used to quantify the plasma concentrations of TAG, glucose 
Table 1. Sequences of primers used in the real-time RT-PCR analysis

\begin{tabular}{|c|c|c|c|c|}
\hline Genes & Symbols & Forward & Reverse & Accession no. \\
\hline $\begin{array}{l}\text { Carnitine } \\
\quad \text { palmitoyltransferase 1a }\end{array}$ & CPT1a & 5'-TGCCTGCCAGTTCCATTAAGC-3' & 5'-GTCTCАСТССТCTTGCCAACAG-3' & NM_031559.2 \\
\hline $\begin{array}{l}\text { Medium-chain acyl } \\
\text { coenzyme A } \\
\text { dehydrogenase }\end{array}$ & $M C A D$ & 5'-GAGGCTACAAGGTCCTGAGAAGTG-3' & 5'-TCTGCTGCTCCGTCAACTCG-3' & NM_016986.2 \\
\hline $\begin{array}{l}\text { Hydroxyacyl-coenzyme } \\
\text { A dehydrogenase }\end{array}$ & $\beta-H A D$ & 5'-СTCСАTGTССТССТСТTССТСTGC-3' & 5'-CAGCCCGCCGCCGATGAC-3' & NM_057186.1 \\
\hline $\begin{array}{l}\text { Pyruvate dehydrogenase } \\
\text { kinase, isozyme } 4\end{array}$ & PDK4 & 5'-GGTGGCGGTGTTCCTCTGAG-3' & 5'-TGAATTGTCCATCACAGGCGTTG-3' & NM_053551.1 \\
\hline Uncoupling protein 3 & UCP3 & 5'-CCGTTAAGCCTTCAGCCTTCC-3' & 5'-CGAGAGTCCATCCTGTCCTTCC-3' & NM 013167.2 \\
\hline$P P A R \alpha$ & PPAR $\alpha$ & 5'-CACGATGCTGTCCTCCTTGATG-3' & $5^{\prime}$-ATGATGTCGCAGAATGGCTTCC- $3^{\prime}$ & NM_013196.1 \\
\hline PPARS & PPAR & 5'-АСТСTССTTTCTTCСTGCCTGTG-3' & 5'-TGTGCTGCTGCTCCTTCTGG-3' & NM_013141.2 \\
\hline $\begin{array}{l}\text { PPAR-gamma, } \\
\text { co-activator } 1 \alpha\end{array}$ & $P G C-1 \alpha$ & 5'-ACACCGCACACATCGCAATTC-3' & 5'-TTCGTCCCTCTTGAGCCTTTCG-3' & NM_031347.1 \\
\hline Myosin heavy chain 7 & Myh7 & 5'-ACAGAGGAAGACAGGAAGAACCTAC-3' & 5'-GGGCTTCACAGGCATCCTTAG-3' & NM 017240.1 \\
\hline $\begin{array}{l}\text { Protein kinase } \\
\quad \text { AMP-activated } \alpha 2\end{array}$ & $A M P K \alpha 2$ & $5^{\prime}$-GATGATGAGGTGGTGGAGCAGAC- $3^{\prime}$ & 5'-CACTGTCTGGCTCTCTCACTGC- ${ }^{\prime}$ & NM_023991.1 \\
\hline Ribosomal 18S & $18 S$ & 5'-GATGCGGCGGCGTTATTC-3' & 5'-CTCCTGGTGGTGCCCTTCC-3' & M11188.1 \\
\hline
\end{tabular}

and cholesterol. The concentrations of NEFA were determined using an assay kit obtained from Wako.

\section{Real-time quantitative $P C R$}

Total RNA from the soleus and EDL muscles was extracted using the TRIzol reagent (Invitrogen) and treated with DNAse (RQ1 DNase, Promega) for $45 \mathrm{~min}$ at $37^{\circ} \mathrm{C}$. Later, $1 \mu \mathrm{g}$ of purified RNA was reverse-transcribed using M-MLV Reverse Transcriptase (Promega) in a total volume of $25 \mu \mathrm{l}$, and the resulting complementary DNA was diluted 40-fold in DNAse and RNAse-free water. Thereafter, $5 \mu l$ of each complementary DNA diluted sample were used as a template for PCR amplification using SYBR Green (Qiagen) as a fluorogenic intercalating dye and the iCycler iQ Real-Time PCR Detection System from Bio-Rad Laboratories. The PCR parameters were as follows: an initial denaturation step of $5 \mathrm{~min}$ at $95^{\circ} \mathrm{C}$, followed by forty cycles of $30 \mathrm{~s}$ at $95^{\circ} \mathrm{C}$ and $30 \mathrm{~s}$ at $60^{\circ} \mathrm{C}$.

Differences in gene expression among the groups were determined using the comparative threshold cycle $\left(C_{\mathrm{t}}\right)$ method as described by Schmittgen \& $\operatorname{Livak}^{(11)}$ and $18 S$ RNA as the housekeeping gene. In brief, the $C_{\mathrm{t}}$ value was automatically determined for all the targeted genes and $18 \mathrm{~S}$ in all the samples and within the same experiment. The relative amount of the different genes normalised to the endogenous expression of $18 S$ was calculated using the following formula: $2^{-\Delta C_{\mathrm{t}}}$, where $\Delta C_{\mathrm{t}}=C_{\mathrm{t}(\text { gene })}-C_{\mathrm{t}(18 S)}$. Fold changes in gene expression due to the different nutritional manipulations were then calculated by dividing the $2^{-\Delta C_{\mathrm{t}}}$ value of each sample by the averaged $2^{-\Delta C_{t}}$ value of the control group. Primers were designed using the information stored in the public GenBank database of the National Center for Biotechnology Information, and their sequences are given in Table 1. The applicability of the $C_{\mathrm{t}}$ method and the use of $18 S$ as the housekeeping gene were first validated by determining how the amplification efficiencies of the different transcripts in relation to those of $18 S, \beta$-actin and glyceraldehyde 3-phosphate dehydrogenase $(G A P D H)$ varied with template dilution. These preliminary experiments indicated that the PCR gave a single product of the expected size for all the genes, as determined by the melting curve analysis and agarose gel electrophoresis (data not shown), and that the amplification efficiency of the target gene was the same independently of the use of $18 S, \beta$-actin or GAPDH as a calibrator (Fig. S1(A), available online). It was then verified whether the expression of $18 S$ as well as that of $\beta$-actin or $G A P D H$ was altered by fasting or by the early exposure to protein restriction (Fig. S1(B), available online). Finally, as shown in Fig. S1(C) (available online), it was confirmed that the pattern of gene expression in the different groups of rats was the same independently of the housekeeping gene used.

\section{Western blot analysis}

Muscle samples were homogenised in 1:8 volume (w/v) of extraction buffer containing 50 mM-HEPES (pH 7.6), $50 \mathrm{~mm}-\mathrm{KCl}$, 50 mm-NaF, 5 mm-Na ${ }_{4} \mathrm{P}_{2} \mathrm{O}_{7}, 1 \mathrm{~mm}$-EDTA, 1 mm-ethylene glycol tetraacetic acid, $1 \mathrm{~mm}$-dithiothreitol, $5 \mathrm{~mm}-\beta$-glycerophosphate, $1 \% \mathrm{NP}-40,1 \mathrm{~mm}-\mathrm{Na}_{3} \mathrm{VO}_{4}$ and a cocktail of protease inhibitors (Sigma, P2714). Homogenates were then centrifuged at $10000 \mathrm{~g}$ at $4^{\circ} \mathrm{C}$ for $10 \mathrm{~min}$. The resulting supernatants were used for Western blot analysis. In brief, $100 \mu \mathrm{g}$ of protein extracts were separated on a $10 \%$ SDS-PAGE gel and transferred onto polyvinylidene difluoride membranes. Non-specific binding was blocked by incubating the membranes for $1 \mathrm{~h}$ at room temperature with 5\% bovine serum albumin dissolved in Tris-buffered saline with Tween-20 (TBST, $10 \mathrm{mm-Tris-HCl,}$ $150 \mathrm{~mm}-\mathrm{NaCl}$ and $0 \cdot 1 \%$ Tween $20 ; \mathrm{pH} 7 \cdot 5$ ). The membranes were then incubated overnight at $4{ }^{\circ} \mathrm{C}$ with a primary antibody against total AMP-activated protein kinase (AMPK) or with a rabbit polyclonal antibody recognising the phosphorylated form of AMPK at Thr172. Both antibodies were used at a dilution of 1:1000 and were purchased from Cell Signaling. To control for protein loading, the same blots were incubated with an anti- $\alpha$-tubulin antibody (Santa Cruz Biotechnology, Inc.) diluted at 1:100. Later, the membranes were incubated for $1 \mathrm{~h}$ 
at room temperature with goat anti-rabbit peroxidaseconjugated secondary antibodies (Jackson ImmunoResearch). Immunoblotted proteins were visualised using the UptiLight ${ }^{\mathrm{TM}}$ HRP blot substrate (Interchim) and analysed using the G:BOX Chemi XL system (Syngene).

\section{Data analysis}

Experimental results are expressed as means with their standard errors. Statistical differences in gene expression under ad libitum feeding conditions between the control and PR rats were assessed using Student's $t$ test. To determine the effects of fasting on plasma metabolite concentrations and gene expression, two-way ANOVA was used followed by Bonferroni's test with maternal diet (control or PR) and nutritional status at killing (ad libitum or fasted) as independent factors. Body weight gain was calculated using the following formula: $\left(\left(W_{n+1}-W_{n} / W_{n}\right)\right) \times 100$, where $W_{n}$ is weight in $g$ on day ' $n$ ' and $\mathrm{W}_{n+1}$ is weight in $\mathrm{g}$ on the next recording day. Differences in body weight were assessed with two-way repeated-measures ANOVA using maternal diet and age as factors. Statistical significance was set at $P<0 \cdot 05$.

\section{Results}

\section{Phenotypic characteristics of the pups and metabolite variations in plasma in response to fasting}

There were no differences in body weight at birth between control and PR pups $(\mathrm{C}=5.21$ (SEM 0.18) g, $n 12 ; \mathrm{PR}=4.86$ (SEM 0.21)g, $n$ 12). However, the offspring born to, and nursed by, PR dams gained significantly less weight during lactation such that at the end of this period they weighed, on average, $58 \%$ less than their control counterparts $(\mathrm{C}=43.38$ ( $\operatorname{sem} 0.46) \mathrm{g}, n 12 ; \mathrm{PR}=19.50$ (SEM 0.66) g, $n 12$, $P<0.0001$; Fig. 1(a)). During the first 2 months after weaning, the PR pups exhibited enhanced body weight gain when compared with control pups (Fig. 1(b)). Nevertheless, in spite of this catch-up growth, they continued to weigh less than control pups until the end of the experimental period (Fig. 1(a)). In addition, the PR pups exhibited less abdominal fat both in absolute terms ( $\mathrm{C}=18.88$ (SEM 2.01) g, $n$ 12; $\mathrm{PR}=11.48$. (SEM 1.32) g, $n 12 ; P<0.01)$ and in proportion to their body weight $(\mathrm{C}=8.26$ (SEM 0.70$) \%, n 12 ; \mathrm{PR}=4.82$ (sem $0 \cdot 32) \%, n 12 ; P<0 \cdot 001)$.

Control and PR rats exhibited the same glucose tolerance as indicated by the identical glucose disappearance rates between 20 and $120 \mathrm{~min}$ after the oral administration of glucose $(\mathrm{C}=308.20(\mathrm{sEM} 32.53) \mathrm{mmol} / 1 \times 120 \mathrm{~min}, n 12$; $\mathrm{PR}=309.80(\operatorname{sem} 41.54) \mathrm{mmol} / \mathrm{l} \times 120 \mathrm{~min}, \quad n$ 12) and the same temporal decrease in plasma glucose concentrations after the glucose load (data not shown). With the exception of a reduced concentration of TAG and insulin, the plasma metabolite profile of the control and PR adult rats was the same under ad libitum feeding conditions (Fig. 2). Both groups also exhibited similar variations in plasma metabolite concentrations in response to a $48 \mathrm{~h}$ fast. Notably, however, fasting reduced the plasma content of insulin in control rats but not in PR rats (Fig. 2).
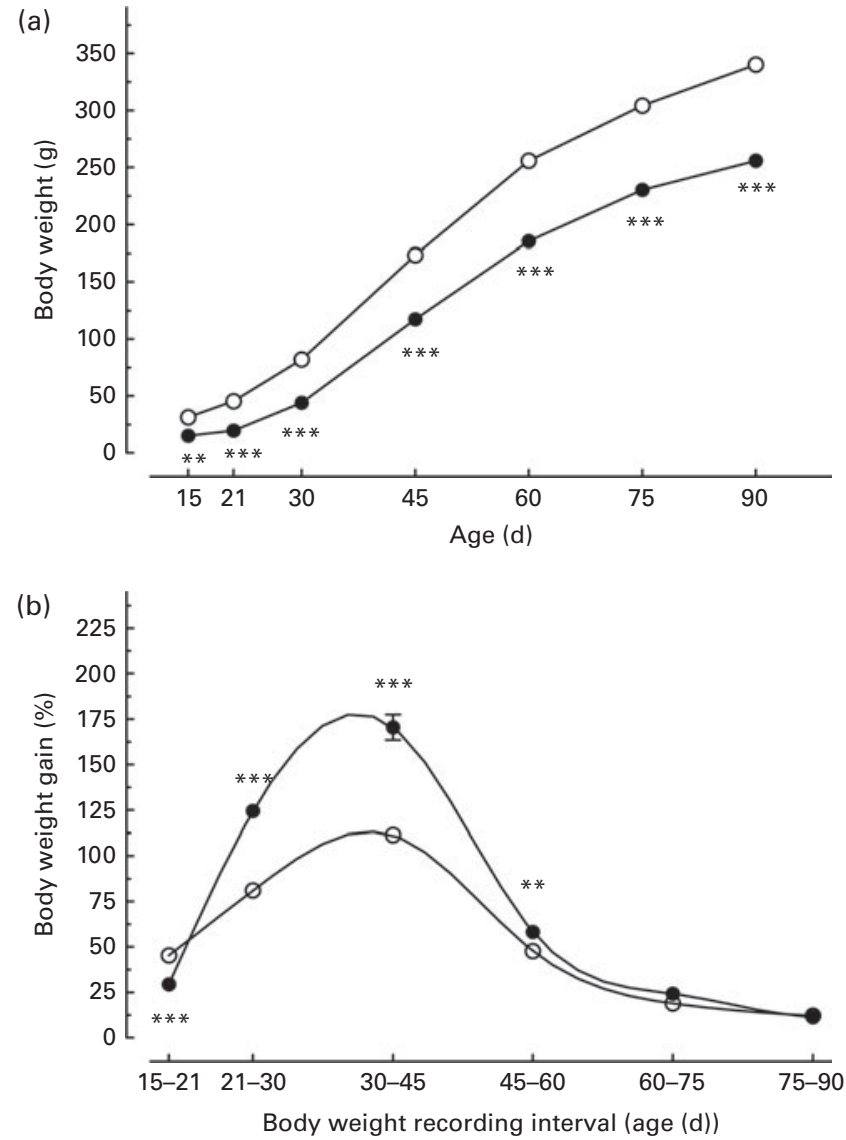

Fig. 1. Evolution of (a) body weight and (b) body weight gain in male rats born to dams fed a control diet (17\% protein) or a low-protein diet $(8 \%$ protein) during gestation and lactation. At weaning, all rats were fed standard chow. Data in (b) correspond to the percentage in body weight gain between the indicated recording periods. Values are means, with their standard errors represented by vertical bars. Mean value was significantly different from that of the control group: ${ }^{\star \star} P<0.01$; ${ }^{\star \star *} P<0.0001$ (two-way repeated-measures ANOVA). $\bigcirc$, Control; $\bullet$, protein restricted.

\section{Gene expression profile under ad libitum feeding conditions}

The mRNA expression of several genes involved in the regulation of fatty acid oxidation was higher in soleus muscle, but not in EDL muscle, of PR rats killed in the fed state than in the muscles of their control counterparts. Thus, the mRNA expression of CPT1a and $M C A D$ was up-regulated in PR rats (Fig. 3). CPT1a is the key enzyme in the carnitine-dependent transport of fatty acids across the mitochondrial inner membrane and MCAD catalyses the first dehydrogenation step in the $\beta$-oxidation of fatty acids. Moreover, the expression of the nuclear receptor PPAR $\alpha$ as well as that of the co-regulator of transcription $P G C-1 \alpha$, which in a coordinated fashion positively regulate the expression of several genes playing a key role in fatty acid oxidation, was increased by more than 2-fold by perinatal protein restriction (Fig. 3). Similar differences in the expression of mRNA encoding the $\alpha 2$ isoform of $A M P K$ were observed between control and PR rats. This kinase is activated by energy depletion and regulates a large number of cellular process that synergistically inhibit protein 

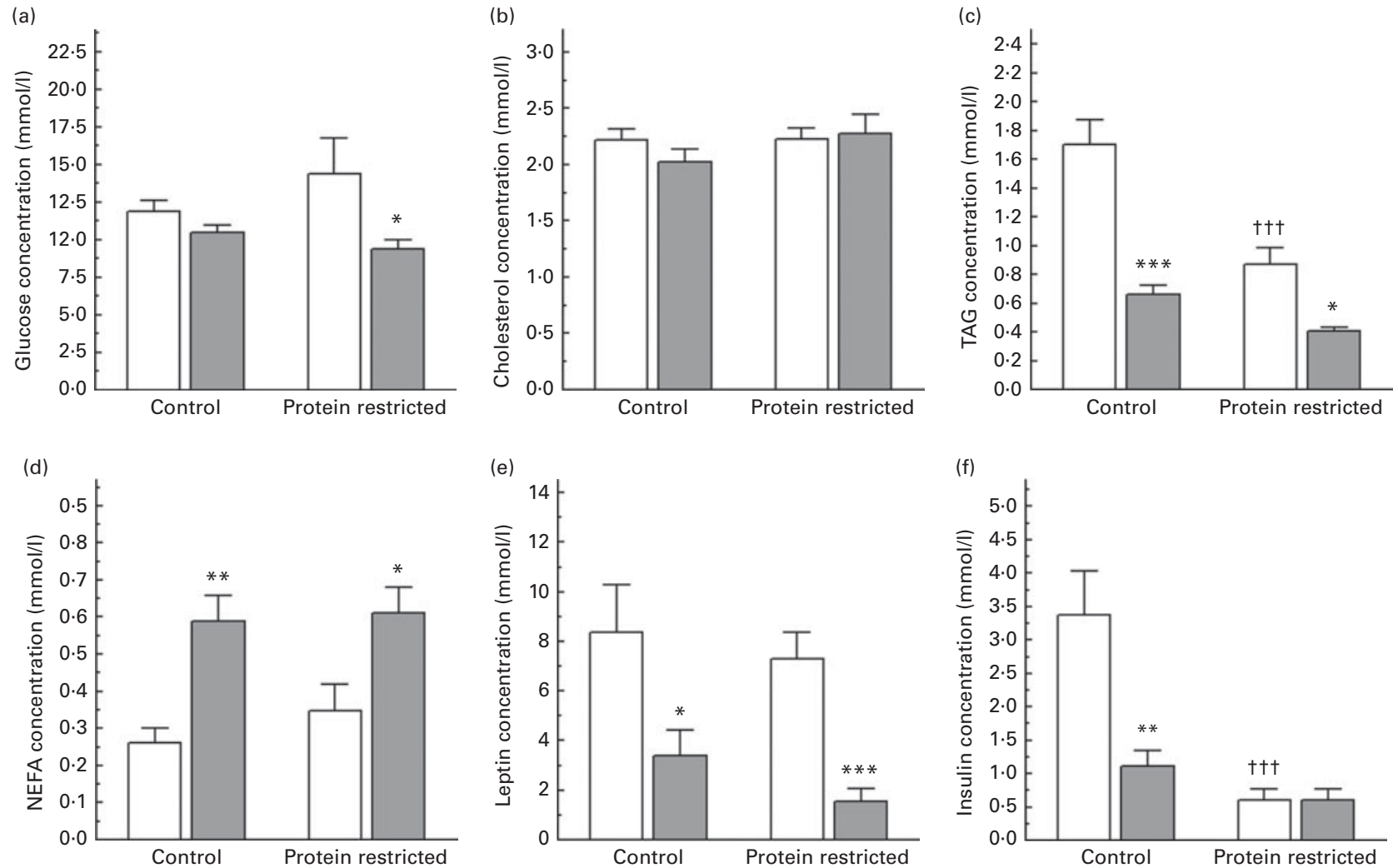

Fig. 2. Metabolite concentrations in the plasma of adult rats born to dams fed a control diet (17\% protein) or a low-protein diet ( $8 \%$ protein) during gestation and lactation. Rats were killed under ad libitum feeding conditions within the first hour of the light cycle or after a $48 \mathrm{~h}$ fast. Data were analysed using two-way ANOVA using maternal diet (control or protein restricted) and nutritional status at killing (ad libitum ( $\square$ ) or fasted $(\square)$ ) as independent factors followed by Bonferroni's test. (a) Glucose, (b) cholesterol, (c) TAG, (d) NEFA, (e) leptin and (f) insulin. Values are means, with their standard errors represented by vertical bars. Mean value was significantly different from that of the ad libitum-fed animals of the same group: ${ }^{\star} P<0.05,{ }^{\star \star} P<0.01,{ }^{\star \star \star} P<0.001$. $\dagger \dagger \dagger$ Mean value was significantly different from that of the ad libitum-fed control group animals $(P<0.001)$.

and lipid biosynthesis and stimulate $\beta$-oxidation, glucose uptake and glycolysis ${ }^{(12)}$. Finally, using Student's $t$ test, an increased expression of $U C P 3$, which exports NEFA from the mitochondrial matrix when fatty acid supply exceeds oxidation $^{(13,14)}$, and a decreased expression of PDK4, which negatively regulates glucose metabolism through the inhibition of the pyruvate dehydrogenase complex, were also detected (Fig. 3). In contrast to these multiple gene expression changes in soleus muscle, the only transcriptional change detected in EDL muscle of ad libitum-fed PR rats was an increased expression of mRNA encoding PPAR $\alpha$ and PPAR $\delta$ nuclear receptors along with a decreased expression of $M C A D$ (Fig. 4).

\section{Gene expression changes in response to fasting}

Both divergent and similar mRNA transcription changes were observed in response to fasting in control and PR rats and these changes differed from one muscle type to the other. Thus, while fasting increased the expression of CPT1a in soleus muscle of control rats, no change in mRNA expression was detected in fasted PR rats when compared with ad libitum-fed PR rats (Fig. 3). Moreover, fasting decreased the expression of $M C A D$ and $\beta$-hydroxyacyl coenzyme A dehydrogenase $(\beta-H A D)$ and $A M P K \alpha 2$ in PR rats but not in control rats (Fig. 3). Similar to MCAD, $\beta$-HAD catalyses several steps of the $\beta$-oxidation of long-chain fatty acids in the mitochondria. In addition, the expression of PDK4 was not affected by fasting neither in control nor PR rats (Fig. 3). Among the genes involved in the transcriptional regulation of metabolism (PGC-1 $\alpha, P P A R \alpha$ and PPAR $\delta$ ), the expression of myosin heavy chain $7(M y b 7)$, which defines the formation of type I (oxidative) fibres, was decreased by fasting in both control and PR rats (Fig. 3). However, it should be noted that in the case of PGC-1 $\alpha$ and PPAR $\alpha$, the differences in gene expression between the ad libitum-fed and fasted control rats reached statistical significance only when the data were analysed using Student's $t$ test.

With regard to the transcriptional changes in EDL muscle, it was found that fasting increased the expression of CPT1a and UCP3 in both groups of rats (Fig. 4). However, fasted PR, but not fasted control rats, exhibited an increased expression of $P D K 4$ and a decreased expression of mRNA encoding PPARS in relation to their ad libitum-fed counterparts (Fig. 4). Moreover, the expression of $M C A D$ was down-regulated by fasting in the control but not in PR rats. Lastly, food deprivation for $48 \mathrm{~h}$ did not alter the mRNA expression of $\beta-H A D, P G C-1 \alpha, A M P K \alpha 2$ and $M H C 1$ neither in control nor in PR rats (Fig. 4). 
(a)

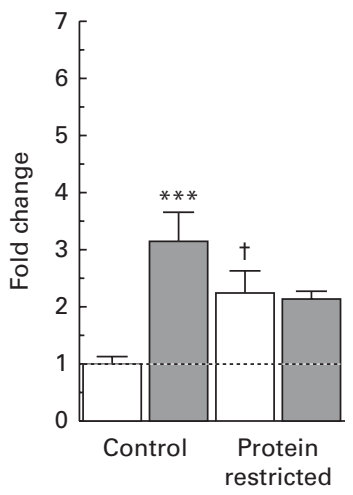

(f)

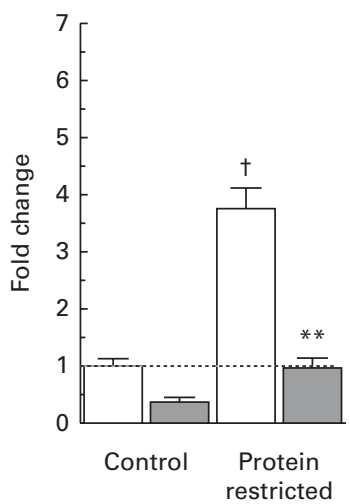

(b)

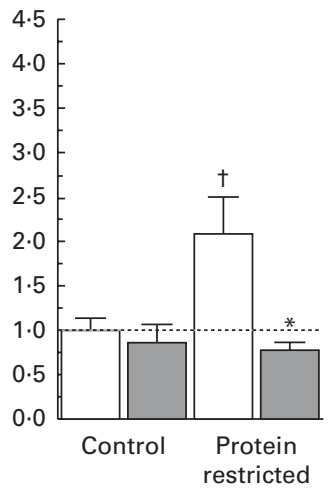

(g)

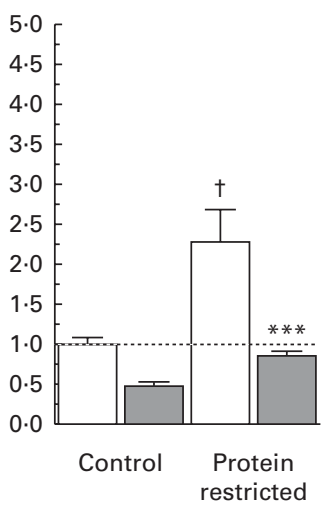

(c)

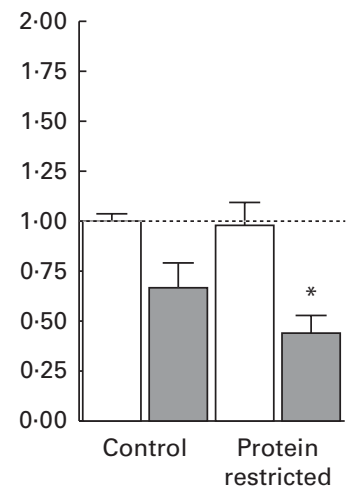

(h)

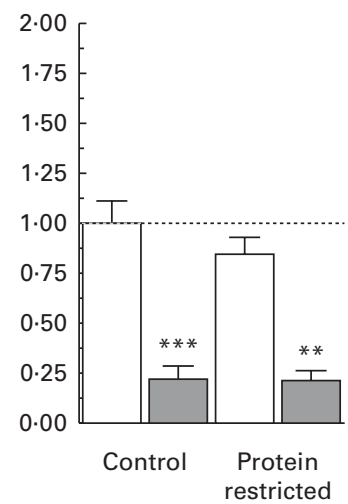

(d)

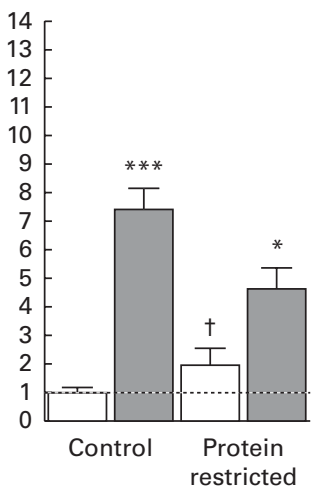

(i)

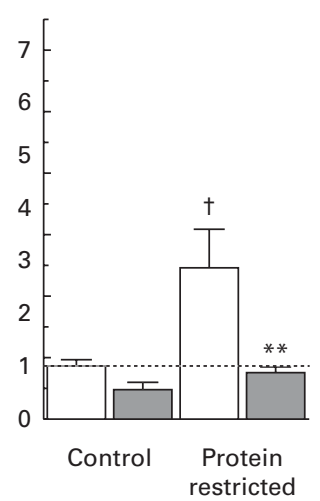

(e)

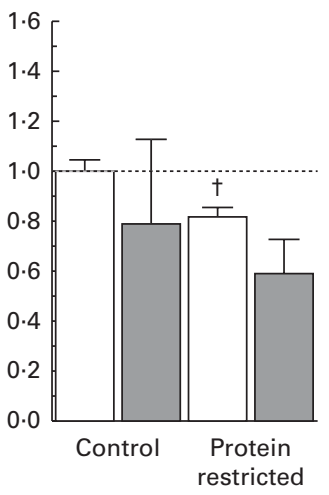

(j)

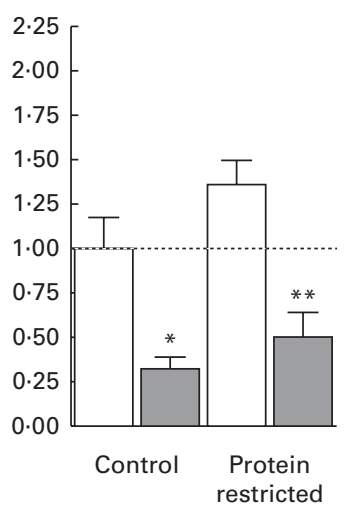

Fig. 3. Gene expression changes in the soleus from control and protein-restricted rats in response to fasting: (a) carnitine palmitoyltransferase 1a; (b) mediumchain acyl coenzyme A dehydrogenase; (c) $\beta$-hydroxyacyl-coenzyme A dehydrogenase; (d) uncoupling protein 3; (e) pyruvate dehydrogenase kinase, isozyme 4; (f) PPAR-gamma, co-activator $1 \alpha$; (g) PPAR $\alpha$; (h) PPAR $\delta$; (i) AMP-activated protein kinase $\alpha 2$; (j) myosin heavy chain class 7 . Variations in gene expression are illustrated in relation to those observed under ad libitum feeding conditions using the expression in fed animals of the control group as a calibrator. ( $\square$ ), Ad libitum; ( $\square$ ), fasted. Values are means, with their standard errors represented by vertical bars. Mean value was significantly different from that of the ad libitum-fed animals of the same group: ${ }^{*} P<0.05,{ }^{* *} P<0.01,{ }^{* \star *} P<0.001$ (two-way ANOVA followed by Bonferroni's test). $\dagger$ Mean value was significantly different from that of the ad libitum-fed control group animals $(P<0.05$; Student's $t$ test).

\section{Effect of maternal protein restriction on the activation of} AMP-activated protein kinase

The nutrient sensor AMPK is activated by energy depletion and once activated regulates a wide range of cellular processes that synergistically inhibit protein and lipid biosynthesis and stimulate $\beta$-oxidation, glucose uptake and glycolysis. Therefore, it was surprising to find no changes in the expression of $A M P K \alpha 2$ in the soleus muscle of control fasted rats. To clearly determine whether AMPK was activated under our experimental conditions, the phosphorylated (activated) form of AMPK at Thr172 was quantified by Western blot analysis. As shown in Fig. 5, PR rats exhibited enhanced phosphorylation levels of AMPK in the fed state in soleus but not in EDL. Moreover, whereas fasting induced a significant activation of AMPK in the soleus muscle of control rats, no change in AMPK phosphorylation levels in response to fasting was detected in PR rats (Fig. 5(a)). Fasting also increased the phosphorylation levels of AMPK in the EDL muscle of control and PR rats, though in control rats this change did not reach statistical significance (Fig. 5(b)).

\section{Discussion}

While reduced energy expenditure and increased consumption of highly energetic foods are important factors that contributing to the current epidemic of obesity, many epidemiological and experimental studies have demonstrated that an unbalanced provision of nutrients during perinatal development increases the risk of developing obesity and the metabolic syndrome in adulthood ${ }^{(15)}$. Several mechanisms have been proposed to account for this enhanced disease risk including altered organ structure ${ }^{(16,17)}$, elevated glucocorticoid levels and endocrine sensitivity ${ }^{(18,19)}$, impaired mitochondrial function ${ }^{(20,21)}$ and altered feeding behaviour ${ }^{(22,23)}$. In the present study, we showed that the metabolic flexibility of skeletal muscle is impaired in adult offspring born to dams fed a low-protein diet during gestation and lactation adding to the detrimental effects of malnutrition during early life.

Skeletal muscle is a heterogeneous tissue that has different types of fibres, namely type I and type II, which differ in acid stability of their myofibrillar ATPase activity and their contractile and metabolic properties ${ }^{(24,25)}$. Type I fibres mainly use fat as an energy substrate, their speed of contraction is 

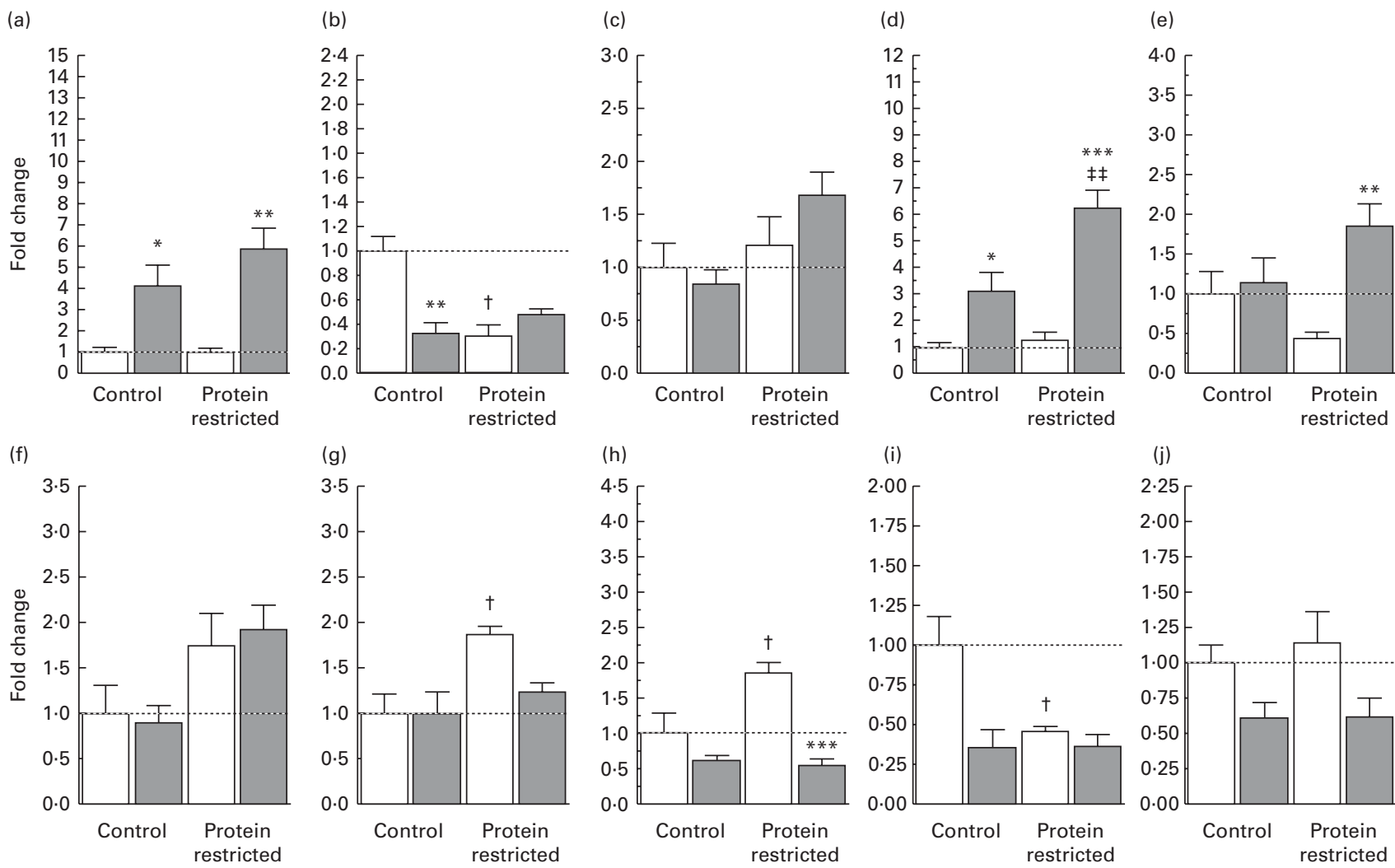

Fig. 4. Gene expression changes in the extensor digitorum longus from control and protein-restricted rats in response to fasting: (a) carnitine palmitoyltransferase 1a; (b) medium-chain acyl coenzyme A dehydrogenase; (c) $\beta$-hydroxyacyl-coenzyme A dehydrogenase; (d) uncoupling protein 3 ; (e) pyruvate dehydrogenase kinase, isozyme 4; (f) PPAR-gamma, co-activator 1 $\alpha$; (g) PPAR $\alpha$; (h) PPAR $\delta$; (i) AMP-activated protein kinase $\alpha 2$; (j) myosin heavy chain class 7 . Variations in gene expression are illustrated in relation to those observed under ad libitum feeding conditions using the expression in fed animals of the control group as a calibrator. ( $\square$ ), Ad libitum; $(\square)$, fasted. Values are means, with their standard errors represented by vertical bars. Mean value was significantly different from that of the ad libitum-fed animals of the same group: ${ }^{*} P<0.05,{ }^{* \star} P<0.01,{ }^{* \star *} P<0.001$ (two-way ANOVA followed by Bonferroni's test). $\dagger$ Mean value was significantly different from that of the ad libitum-fed control group animals $(P<0.05$; Student's $t$ test). $\ddagger \ddagger$ Mean value was significantly different from that of the fasted control group animals $(P<0.01)$.

relatively slow and they are resistant to fatigue. In contrast, type II fibres use glucose as an energy source, contract quickly and do not have a high resistance to fatigue. On the basis of their $\mathrm{pH}$ liability, type II fibres are further categorised into type IIa, which possess a mixture of glycolytic and oxidative properties, and type IIb, which are exclusively glycolytic ${ }^{(26)}$ The soleus muscle consists of only type I and type IIa fibres, whereas the EDL muscle mainly consists of type IIa and type IIb fibres. Consequently, because of its high oxidative capacity, the soleus muscle tends to use NEFA as energy substrates, whereas the EDL muscle has far higher basal rates of glycolysis and gluconeogenesis. Moreover, these two muscles use different mechanisms to cope with starvation-induced elevated NEFA concentrations. In fact, the soleus muscle disposes of excess NEFA by oxidation, whereas the EDL muscle neutralises NEFA by re-esterification ${ }^{(27)}$. Therefore, to determine the effects of early malnutrition on the metabolic flexibility of skeletal muscle, we investigated the expression of several genes involved in lipid metabolism and mitochondrial function in the soleus and EDL muscles as they possess opposite metabolic properties.

Under ad libitum feeding conditions, PR rats exhibited increased $A M P K$ gene expression and phosphorylation levels in soleus muscle along with an enhanced mRNA expression of several genes encoding key proteins favouring fatty acid oxidation including CPT1a, PGC-1 $\alpha$, UCP3 and PPAR $\alpha$. In contrast, there was an increased mRNA expression of PPAR $\alpha$ and PPAR $\delta$ along with a decreased gene expression of $M C A D$ in EDL muscle, indicating that, in addition to having divergent metabolic properties, these two muscles are differentially affected by metabolic programming.

Increased $\beta$-oxidation ${ }^{(28)}$ and overexpression of $C P T 1^{(29)}$ in skeletal muscle cells have been shown to improve insulin resistance. The gene expression data obtained in the present study, along with the observation that PR rats also had reduced body weight and decreased plasma concentrations of TAG, indicate that early protein restriction induces a longlasting metabolic state favouring lipid catabolism and insulin function. This observation seems to be at odds with the thrifty phenotype hypothesis stating that a poor nutritional environment during early life increases the risk of developing the metabolic syndrome in adulthood ${ }^{(3)}$. However, enhanced capacity for fat oxidation in muscle is not in itself predictive of a healthy phenotype. In fact, transgenic mice in which increased fatty acid oxidation is induced by muscle-specific overexpression of PPAR $\alpha$ or $P G C-1 \alpha$ are more susceptible 
(a)
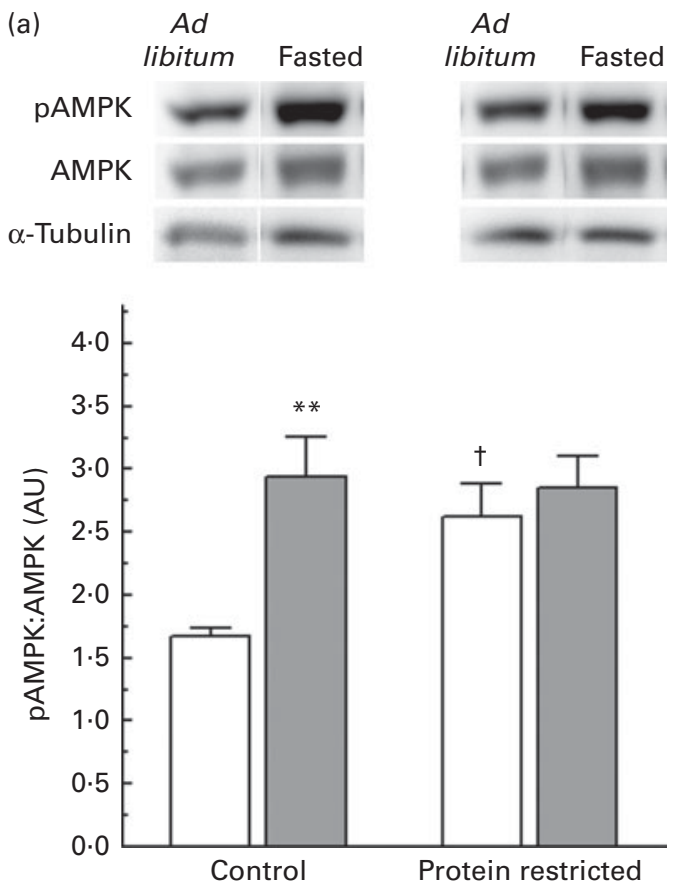

(b)
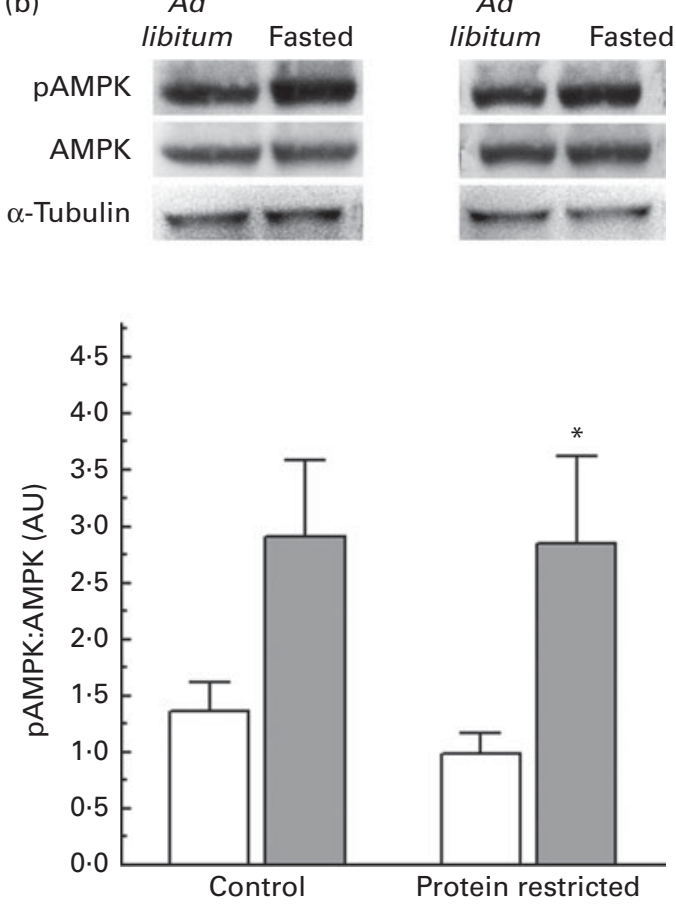

Fig. 5. Changes in AMP-activated protein kinase (AMPK) activity in response to fasting in the (a) soleus muscle and (b) extensor digitorum longus muscle of adult rats born to control or protein-restricted dams. Each bar corresponds to the ratio of phosphorylated levels:non-phosphorylated levels of AMPK determined by Western blot analysis. ( $\square$ ), Data obtained from rats killed under ad libitum feeding conditions. ( $\square$ ), Data obtained from rats killed after a fasting period of $48 \mathrm{~h}$. Mean value was significantly different from that of the ad libitum-fed animals of the same group: ${ }^{*} P<0.05$, ${ }^{\star *} P<0.01$ (two-way ANOVA followed by Bonferroni's test). $†$ Mean value was significantly different from that of the ad libitum-fed control group animals $(P<0.05$; two-way ANOVA followed by Bonferroni's test). pAMPK, phospho AMPK; AU, arbitrary units. to diet-induced obesity and diabetes ${ }^{(30)}$. Moreover, high-fat diet feeding increases the lipid oxidation capacity of skeletal muscle through the up-regulation of several key genes regulating mitochondrial biogenesis ${ }^{(31)}$. On the basis of these results, it has been suggested that incomplete fatty acid oxidation and accumulation of $\beta$-oxidative intermediates resulting from the mismatch between lipid overload and energy needs are the primary biochemical defects underlying insulin resistance ${ }^{(1,32)}$. In this respect, it is interesting to note that ad libitum-fed PR rats exhibited an enhanced expression of CPT1a and UCP3 in the soleus muscle without any modification in the expression of mRNA encoding $\beta-H A D$. This transcriptional profile is indicative of an enhanced flux of lipids into the mitochondria without a concomitant increase in fatty acid oxidation. This would lead to increased export of fatty acid anions outside the mitochondrial matrix, which might result in the accumulation of lipids in skeletal muscle.

Fasting is a nutritional challenge that leads to rapid metabolic adaptations of skeletal muscle that result in the shift from glucose to fatty acid oxidation as the source of fuel. This phenomenon involves a complex mechanism of transcriptional regulation that includes the nuclear receptors $P P A R \alpha$ and PPAR $\delta$, the co-transcriptional regulator $P G C-1 \alpha$ and the nutrient sensors sirtuin 1 and $\mathrm{AMPK}^{(33-35)}$. The two latter factors, which are activated under physiological conditions that require a large amount of energy such as nutritional restriction or exercise, induce the expression of $P G C-1 \alpha$ and directly enhance its activity through deacetylation (sirtuin 1) or phosphorylation (AMPK) ${ }^{(36)}$. The expression of $P G C-1 \alpha$ can also be enhanced via the nuclear receptor $\operatorname{PPAR} \delta^{(37)}$ and a Ca-dependent signalling pathway involving the activation of the transcription factor C-AMP response element-binding protein $(\mathrm{CREB})^{(38)}$. Once activated, PGC- $1 \alpha$ facilitates the expression of several genes involved in fatty acid oxidation and mitochondrial function by forming a complex with the nuclear receptor PPAR $\alpha^{(39)}$ and, simultaneously, regulates the reprogramming of fast-twitch, glycolytic fibres (type II) into slow-twitch, oxidative fibres (type I) through the co-activation of myosin enhancer factor $2^{(40)}$.

The transcriptional responses of skeletal muscle to fasting are time dependent. Thus, the expression of PPAR $\delta$ and $P G C-1 \alpha$ is initially up-regulated by food deprivation, but after a $48 \mathrm{~h}$ fast, it decreases below control levels ${ }^{(41)}$. In agreement with these results, we observed that a $48 \mathrm{~h}$ starvation period reduced the expression of these two transcription factors as well as that of PPAR $\alpha$ in the soleus muscle of both control and PR rats. However, we found a clear difference in the gene expression profiles of CPT1a, MCAD and $\beta-H A D$ between control and PR rats in response to fasting. In fact, the mRNA levels of the former gene were increased by starvation in control rats but not in PR rats. Moreover, whereas fasted PR rats exhibited a reduced expression of $M C A D$ and $\beta-H A D$ in relation to their ad libitum-fed counterparts, starvation induced no change in the expression of these genes in control rats. Similarly, the activation of AMPK, as measured by the phosphorylation of its $\alpha$-subunit at Thr172, was increased by fasting in control but not in PR rats. Altogether, these gene expression changes are indicative of 
impaired fatty acid uptake and oxidation in response to fasting in the soleus muscle of PR rats. Furthermore, as expected from their divergent metabolic properties, the soleus and EDL muscles reacted differently to food deprivation and their metabolic flexibility was differentially affected by early protein restriction. Thus, while starvation down-regulated the expression of the transcriptional regulators of lipid metabolism (PPAR $\alpha, P P A R \delta$ and $P G C-1 \alpha)$ in the soleus muscle of both control and PR rats, this effect was observed only in the EDL muscle of PR rats. In addition, in sharp contrast to its effects in soleus muscle, fasting induced a gene expression profile indicative of enhanced fatty acid $\beta$-oxidation and reduced glycolysis in EDL muscle of PR rats. Collectively, these results show that fasting alters the metabolic properties of skeletal muscle in a very fine and selective manner.

Kelley et $a l .{ }^{(1)}$ were the first to propose that the metabolic inflexibility of skeletal muscle, defined as decreased fatty acid oxidation under fasting conditions or reduced capacity to switch from lipid oxidation to glucose uptake and catabolism during the transition from fasting to feeding, is associated with obesity and diabetes and predicts the severity of insulin resistance. Further studies have corroborated that skeletal muscle of obese or diabetic patients exhibits decreased fatty acid oxidation during fasting or exercise as well as reduced capacity to shift between lipid and glucose oxidation under insulin-stimulating conditions ${ }^{(42,43)}$. However, it has not been determined whether metabolic inflexibility is an additional manifestation of obesity and diabetes or whether it is an underlying cause of these metabolic disorders. In the present study, we showed that 4-month-old rats born to PR dams exhibit an altered gene expression pattern in skeletal muscle in response to fasting. The fact that, at this age, these animals did not exhibit any sign of obesity or glucose intolerance suggests that metabolic inflexibility might be the cause and not the consequence of obesity and diabetes. Interestingly, early protein restriction differentially affected the responses of the soleus and EDL muscles to fasting, further indicating that the metabolic inflexibility of skeletal muscle might be fibre type specific.

In summary, the results of the present study indicate that under ad libitum feeding conditions adult offspring born to PR dams exhibit a gene expression pattern in skeletal muscle favouring the transport of long-chain fatty acids into the mitochondrial matrix without a concomitant increase in fatty acid oxidation. Moreover, the transcriptional network regulating the metabolic switch from carbohydrate to lipid oxidation during fasting was clearly altered in the PR rats, which otherwise exhibit a normal metabolic state. This observation suggests that impaired metabolic flexibility precedes and may underpin the development of obesity and insulin resistance induced by maternal protein restriction. Given that the deleterious effects of early malnutrition develop with age, further studies are warranted to determine how the herein reported impaired metabolic plasticity of skeletal muscle might contribute to insulin resistance and obesity.

\section{Supplementary material}

To view supplementary material for this article, please visit http://dx.doi.org/10.1017/S0007114514000865

\section{Acknowledgements}

The authors cordially thank Dr Elizabeth do Nascimento from the Nutrition Department of the Federal University of Pernambuco for preparing the experimental diets.

R. d. S. A. was the recipient of a doctoral fellowship from the Brazilian Coordenação de Aperfeiçoamento de Pessoal de Nível Superior (CAPES). O. G.-Q. and G. P.-G. were the recipients of, respectively, a doctoral and a post-doctoral fellowship from the Mexican Consejo Nacional de Ciencia y Tecnología. The present study was partially supported by CAPES-COFECUB (Action Number Me 657/09). The funders had no role in the design and analysis of the study or in the writing of this article.

The authors' contributions are as follows: F. B.-J. conceived the study, designed the experiments, analysed the data and wrote the manuscript; R. d. S. A. carried out the experiments and contributed to the writing of the manuscript; O. G.-Q. and G. P.-G. conducted the experiments; R. M.-d.-C. contributed to the design of the experiments.

The authors have no competing interests to declare.

\section{References}

1. Kelley DE, Goodpaster B, Wing RR, et al. (1999) Skeletal muscle fatty acid metabolism in association with insulin resistance, obesity, and weight loss. Am J Physiol 277, E1130-E1141.

2. Storlien L, Oakes ND \& Kelley DE (2004) Metabolic flexibility. Proc Nutr Soc 63, 363-368.

3. Hales CN \& Barker DJ (1992) Type 2 (non-insulindependent) diabetes mellitus: the thrifty phenotype hypothesis. Diabetologia 35, 595-601.

4. Selak MA, Storey BT, Peterside I, et al. (2003) Impaired oxidative phosphorylation in skeletal muscle of intrauterine growth-retarded rats. Am J Physiol Endocrinol Metab 285, E130-E137.

5. Ozanne SE, Wang CL, Coleman N, et al. (1996) Altered muscle insulin sensitivity in the male offspring of proteinmalnourished rats. Am J Physiol Endocrinol Metab 271, E1128-E1134.

6. Ozanne SE, Olsen GS, Hansen LL, et al. (2003) Early growth restriction leads to down regulation of protein kinase $C$ zeta and insulin resistance in skeletal muscle. J Endocrinol 177, $235-241$.

7. Mallinson JE, Sculley DV, Craigon J, et al. (2007) Fetal exposure to a maternal low-protein diet during midgestation results in muscle-specific effects on fibre type composition in young rats. Br J Nutr 98, 292-299.

8. Toscano AE, Manhaes-de-Castro R \& Canon F (2008) Effect of a low-protein diet during pregnancy on skeletal muscle mechanical properties of offspring rats. Nutrition $\mathbf{2 4}$, 270-278.

9. Bayol S, Jones D, Goldspink G, et al. (2004) The influence of undernutrition during gestation on skeletal muscle cellularity and on the expression of genes that control muscle growth. Br J Nutr 91, 331-339.

10. Orozco-Solis R, Lopes de Souza S, Barbosa Matos RJ, et al. (2009) Perinatal undernutrition-induced obesity is 
independent of the developmental programming of feeding. Physiol Behav 96, 481-492.

11. Schmittgen TD \& Livak KJ (2008) Analyzing real-time PCR data by the comparative CT method. Nat Protoc 3, 1101-1108.

12. Lage R, Dieguez C, Vidal-Puig A, et al. (2008) AMPK: a metabolic gauge regulating whole-body energy homeostasis. Trends Mol Med 14, 539-549.

13. Abe T, Mujahid A, Sato K, et al. (2006) Possible role of avian uncoupling protein in down-regulating mitochondrial superoxide production in skeletal muscle of fasted chickens. FEBS Lett 580, 4815-4822.

14. Himms-Hagen J \& Harper ME (2001) Physiological role of UCP3 may be export of fatty acids from mitochondria when fatty acid oxidation predominates: an hypothesis. Exp Biol Med 226, 78-84.

15. Ozanne SE \& Constancia M (2007) Mechanisms of disease: the developmental origins of disease and the role of the epigenotype. Nat Clin Pract Endocrinol Metab 3, 539-546.

16. Merezak S, Reusens B, Renard A, et al. (2004) Effect of maternal low-protein diet and taurine on the vulnerability of adult Wistar rat islets to cytokines. Diabetologia 47, 669-675.

17. Vehaskari VM, Aviles DH \& Manning J (2001) Prenatal programming of adult hypertension in the rat. Kidney Int 59, 238-245.

18. Cottrell EC, Holmes MC, Livingstone DE, et al. (2012) Reconciling the nutritional and glucocorticoid hypotheses of fetal programming. FASEB J 26, 1866-1874.

19. Fowden AL \& Forhead AJ (2009) Hormones as epigenetic signals in developmental programming. Exp Physiol 94, 607-625.

20. Reusens B, Theys N \& Remacle C (2011) Alteration of mitochondrial function in adult rat offspring of malnourished dams. World J Diabetes 15, 149-157.

21. Leduc L, Levy E, Bouity-Voubou M, et al. (2010) Fetal programming of atherosclerosis: possible role of the mitochondria. Eur J Obstet Gynecol Reprod Biol 149, 127-130.

22. Vickers MH, Breier BH, Cutfield WS, et al. (2000) Fetal origins of hyperphagia, obesity, and hypertension and postnatal amplification by hypercaloric nutrition. Am J Physiol Endocrinol Metab 279, E83-E87.

23. Bellinger L \& Langley-Evans SC (2005) Fetal programming of appetite by exposure to a maternal low-protein diet in the rat. Clin Sci 109, 413-420.

24. Punkt K, Naupert A \& Asmussen G (2004) Differentiation of rat skeletal muscle fibres during development and ageing. Acta Histochem 106, 145-154.

25. Schiaffino S \& Reggiani C (2011) Fiber types in mammalian skeletal muscles. Physiol Rev 91, 1447-1531.

26. Brooke MH \& Kaiser KK (1970) Three myosin adenosine triphosphatase systems: the nature of their $\mathrm{pH}$ lability and sulfhydryl dependence. J Histochem Cytochem 18, 670-672.

27. Neumann-Haefelin C, Beha A, Kuhlmann J, et al. (2004) Muscle-type specific intramyocellular and hepatic lipid metabolism during starvation in Wistar rats. Diabetes 53, 528-534.

28. Perdomo G, Commerford SR, Richard A-MT, et al. (2004) Increased $\beta$-oxidation in muscle cells enhances insulinstimulated glucose metabolism and protects against fatty acid-induced insulin resistance despite intramyocellular lipid accumulation. J Biol Chem 279, 27177-27186.

29. Bruce CR, Hoy AJ, Turner N, et al. (2009) Overexpression of carnitine palmitoyltransferase- 1 in skeletal muscle is sufficient to enhance fatty acid oxidation and improve high-fat diet-induced insulin resistance. Diabetes 58, 550-558.

30. Choi CS, Befroy DE, Codella R, et al. (2008) Paradoxical effects of increased expression of PGC- $1 \alpha$ on muscle mitochondrial function and insulin-stimulated muscle glucose metabolism. Proc Natl Acad Sci 105, 19926-19931.

31. Turner N, Bruce CR, Beale SM, et al. (2007) Excess lipid availability increases mitochondrial fatty acid oxidative capacity in muscle: evidence against a role for reduced fatty acid oxidation in lipid-induced insulin resistance in rodents. Diabetes 56, 2085-2092.

32. Muoio Deborah M \& Neufer PD (2012) Lipid-induced mitochondrial stress and insulin action in muscle. Cell Metab 15, 595-605.

33. Bassel-Duby R \& Olson EN (2006) Signaling pathways in skeletal muscle remodeling. Annu Rev Biochem 75, 19-37.

34. Jørgensen SB, Richter EA \& Wojtaszewski JF (2006) Role of AMPK in skeletal muscle metabolic regulation and adaptation in relation to exercise. $J$ Physiol 574, 17-31.

35. Rodgers JT, Lerin C, Gerhart-Hines Z, et al. (2008) Metabolic adaptations through the PGC-1 alpha and SIRT1 pathways. FEBS Lett 582, 46-53.

36. Cantó C \& Auwerx J (2009) PGC-1[alpha], SIRT1 and AMPK, an energy sensing network that controls energy expenditure. Curr Opin Lipidol 20, 98-105.

37. Schuler M, Ali F, Chambon C, et al. (2006) PGC1 $\alpha$ expression is controlled in skeletal muscles by PPAR $\beta$, whose ablation results in fiber-type switching, obesity, and type 2 diabetes. Cell Metab 4, 407-414.

38. Chowanadisai W, Bauerly KA, Tchaparian E, et al. (2010) Pyrroloquinoline quinone stimulates mitochondrial biogenesis through CAMP response element-binding protein phosphorylation and increased PGC- $1 \alpha$ expression. $J$ Biol Chem 285, $142-152$.

39. Vega RB, Huss JM \& Kelly D (2000) The coactivator PGC-1 cooperates with peroxisome proliferator-activated receptor $\alpha$ in transcriptional control of nuclear genes encoding mitochondrial fatty acid oxidation enzymes. Mol Cell Biol 20, 1868-1876.

40. Lin J, Wu H, Tarr PT, et al. (2002) Transcriptional co-activator PGC-1[alpha] drives the formation of slow-twitch muscle fibres. Nature 418, 797-801.

41. de Lange P, Farina P, Moreno M, et al. (2006) Sequential changes in the signal transduction responses of skeletal muscle following food deprivation. FASEB J 20, 2579-2581.

42. Blaak EE, van Aggel-Leijssen DP, Wagenmakers AJ, et al. (2000) Impaired oxidation of plasma-derived fatty acids in type 2 diabetic subjects during moderate-intensity exercise. Diabetes 49, 2102-2107.

43. Thyfault JP, Rector RS \& Noland RC (2006) Metabolic inflexibility in skeletal muscle: a prelude to the cardiometabolic syndrome? J Cardiometab Syndr 1, 184-189. 